\title{
Projeto da reologia de concretos refratários zero-cimento no sistema $\mathrm{Al}_{2} \mathrm{O}_{3}-\mathrm{SiC}-\mathrm{C}$
}

\section{(Rheological design of zero-cement refractory castables in the $\mathrm{Al}_{2} \mathrm{O}_{3}$-SiC-C system)}

\author{
I. R. de Oliveira, A. R. Studart, V. C. Pandolfelli \\ Universidade Federal de S. Carlos, DEMa \\ Rod. Washington Luiz, km 235, C.P. 676, 13565-905, S. Carlos, SP \\ ivone@iris.ufscar.brevicpando@power.ufscar.br
}

\begin{abstract}
Resumo
Concretos refratários no sistema $\mathrm{Al}_{2} \mathrm{O}_{3}$-SiC-C vêm sendo utilizados principalmente no revestimento de canais de corrida de altos-fornos. A associação dessas matérias-primas tem elevado o desempenho dos concretos refratários frente às severas condições de trabalho, devido ao aumento da resistência ao choque térmico e ao ataque por escória e metal fundido. Apesar disso, o nível de conhecimento científico sobre a dispersão das partículas desse sistema multifásico é limitado. Neste trabalho, suspensões representativas da matriz de concretos refratários zero-cimento no sistema $\mathrm{Al}_{2} \mathrm{O}_{3}-\mathrm{SiC}-\mathrm{C}$ foram preparadas para a avaliação da eficiência de aditivos na dispersão simultânea de diferentes matérias-primas, bem como para a análise do comportamento reológico da matriz em função do $\mathrm{pH}$ e teor de aditivo. Os ácidos poliacrílico e cítrico e um surfactante não iônico foram usados como dispersantes. Os valores de viscosidade aparente e tensão de escoamento obtidos foram usados para construir mapas de estabilidade da matriz. Medidas de $\mathrm{pH}$ e fluidez de concretos preparados com diferentes teores de aditivo mostraram que as condições iniciais de $\mathrm{pH}$ não foram correspondentes à região ótima de dispersão estabelecida pelos mapas de estabilidade. $\mathrm{O}$ uso de altos teores de ácido cítrico, bem como, o deslocamento do $\mathrm{pH}$ do concreto na direção da região ótima de dispersão foram eficientes para otimizar a sua fluidez.
\end{abstract}

Palavras-chave: reologia, concreto refratário, choque térmico.

\section{INTRODUÇÃO}

Dentre os refratários empregados na produção de aços, os concretos se destacam principalmente por possibilitar a automação da etapa de aplicação do revestimento refratário e pelo desempenho frente às severas condições de trabalho. Entretanto, a necessidade de se produzir aços de alta qualidade com baixo custo, vem exigindo melhorias na qualidade dos concretos refratários, com o intuito de aumentar o tempo de vida útil desses materiais cerâmicos.

Concretos refratários no sistema $\mathrm{Al}_{2} \mathrm{O}_{3}-\mathrm{SiC}-\mathrm{C}$ são utilizados principalmente nos canais de corrida de altos-fornos. Tais refratários

\begin{abstract}
Refractory castables in the $\mathrm{Al}_{2} \mathrm{O}_{3}-\mathrm{SiC}-\mathrm{C}$ system have been used mainly as linings for blast furnace runners. The association of these raw materials has enabled the development of high quality refractory castables due to improvements on their resistance to thermal shock, slag and metal corrosion. Nevertheless, the scientific knowledge regarding particle dispersion in this system is quite limited. In this work, representative suspensions of the matrix of $\mathrm{Al}_{2} \mathrm{O}_{3}$-SiC-C refractory castables were prepared in order to evaluate the efficiency of additives on the dispersion state of different raw materials and analyze the matrix rheological behavior as a function of $\mathrm{pH}$ and additive content. The polyacrylic and citric acids and a non-ionic surfactant were utilized as dispersants. The apparent viscosity and yield stress data obtained from the suspension rheological evaluation were used to elaborate matrix stability maps. Initial $\mathrm{pH}$ and flowability results for castables with different additive contents revealed that initial $\mathrm{pH}$ conditions were not coincident with the optimum dispersing region established by the stability maps. A shift of the castable $\mathrm{pH}$ value towards the optimum dispersing region was shown to be an effective approach to increase castable flowability.
\end{abstract}

Keywords: rheological, refractory castables, thermal shock. são considerados compósitos singulares onde cada matéria-prima contribui com suas características típicas. A alumina $\left(\mathrm{Al}_{2} \mathrm{O}_{3}\right)$ apresenta alta refratariedade, alta dureza, alta resistência ao ataque químico e elevado ponto de fusão [1]. O carbeto de silício ( $\mathrm{SiC})$, por sua vez, exibe alta dureza, resistência mecânica à alta temperatura, boa resistência ao choque térmico, coeficiente de expansão térmico relativamente baixo e alta condutividade térmica [2]. O grafite (C) contribui para a redução da expansão térmica, e para o aumento da condutividade térmica, da resistência ao choque térmico, da energia de fratura, assim como da resistência ao ataque por escória decorrente da sua inferior molhabilidade por óxidos fundidos $[3,4]$. 
Uma vez estabelecidas as matérias-primas a serem utilizadas, definindo assim os aspectos químicos, é através do projeto da microestrutura e do processamento que será determinado o desempenho do material refratário. Com o crescente interesse no desenvolvimento de refratários avançados, o domínio do processamento destes materiais é de suma importância, pois permite um controle mais efetivo sobre os defeitos que podem comprometer a homogeneidade microestrutural e o desempenho do produto cerâmico.

A otimização da reologia e do empacotamento de partículas é indispensável para a produção de concretos refratários avançados. Para tanto, um controle simultâneo da distribuição de tamanho de partículas e do estado de dispersão da matriz deve ser efetuado. Os concretos refratários podem ser visualizados como uma suspensão aquosa com alta concentração de sólidos onde a adequada dispersão da matriz (fração de partículas $<\sim 100 \mu \mathrm{m}$ ) possibilita a obtenção de superiores valores de fluidez [5]. Simultaneamente, a dispersão possibilita que a distribuição de partículas projetada possa ser realmente alcançada, aumentando a densidade de empacotamento e reduzindo a quantidade de água do concreto. Portanto, o comportamento reológico e as características de aplicação dos concretos são fortemente influenciados pelo estado de dispersão das partículas [6].

Vários aditivos orgânicos, tais como poliacrilatos e ácido cítrico, vêm sendo utilizados com sucesso para promover a dispersão de suspensões de $\mathrm{Al}_{2} \mathrm{O}_{3}$ com teores relativamente baixos de sólidos $[7,8]$. Em suspensões de $\mathrm{Al}_{2} \mathrm{O}_{3}$ contendo uma segunda fase, a dispersão tem sido obtida utilizando-se aditivos que promovam cargas elétricas de mesmo sinal na superfície das partículas [9]. No caso do sistema $\mathrm{Al}_{2} \mathrm{O}_{3}-\mathrm{SiC}-\mathrm{C}$, é esperado que a presença de grafite dificulte o processamento da suspensão, em virtude da sua baixa molhabilidade pela água. Agentes ativos na superfície vem sendo utilizados para melhorar a molhabilidade e a dispersão das partículas de grafite em suspensão aquosa, através da redução da energia superficial da interface grafite/água [10].

Dentro desse contexto, o objetivo desse trabalho é avaliar as condições ideais de dispersão de suspensões concentradas contendo $\mathrm{Al}_{2} \mathrm{O}_{3}, \mathrm{SiC}$ e $\mathrm{C}$ (p.e., teor de aditivo e $\mathrm{pH}$ ), visando otimizar a reologia de concretos refratários zero-cimento contendo essas matérias-primas.

\section{MATERIAIS E MÉTODOS}

Uma vez que partículas com tamanho $<100 \mu \mathrm{m}$ e com área específica $>1 \mathrm{~m}^{2} / \mathrm{g}$ afetam diretamente a dispersão de concretos refratários, matérias-primas com tais características foram selecionadas para o estudo da dispersão de concretos refratários no sistema $\mathrm{Al}_{2} \mathrm{O}_{3}-\mathrm{SiC}-\mathrm{C}$. $\mathrm{Na}$ Tabela I são apresentadas algumas características das matérias-primas utilizadas: alumina calcinada A-3000FL, carbeto de silício micronizado (SiC Powders, grão tipo 1000) e grafite sintético (Unicarbo 100).

Suspensões representativas da matriz de concretos foram preparadas como apresentado na Tabela II. Utilizando-se tais suspensões, avaliou-se a eficiência dos aditivos na dispersão simultânea de diferentes matérias-primas, bem como o comportamento reológico da matriz.

Os aditivos utilizados foram um sal de ácido poliacrílico (Darvan$7 \mathrm{~S}$, Vanderbilt) com um peso molecular médio de $2500 \mathrm{~g} / \mathrm{mol}$, ácido cítrico (Labsynth, 192 g/mol) e um surfactante não-iônico,
Tabela I - Matérias-primas utilizadas e suas características. [Table I - Characteristics of the raw materials.]

\begin{tabular}{lccc}
\hline & \multicolumn{3}{c}{ Matérias-primas } \\
\cline { 2 - 4 } & Alumina $^{(1)}$ & $\begin{array}{c}\text { Carbeto } \\
\text { de silício }\end{array}$ & Grafite $^{(1)}$ \\
\hline pureza $(\%)$ & 99,85 & 94,26 & 99,20 \\
\hline área específica $\left(\mathrm{m}^{2} / \mathrm{g}\right)$ & 2,98 & 5,22 & 3,80 \\
\hline diâmetro médio & 2,8 & 5,0 & 44,6 \\
de partícula $(\mu \mathrm{m})$ & & & \\
\hline
\end{tabular}

(1) Alcoa Alumínio S/A.

(2) Unimetal- Indústria, Comércio e Empreendimentos Ltda.

Tabela II - Composição da matriz de concreto zero-cimento no sistema $\mathrm{Al}_{2} \mathrm{O}_{3}-\mathrm{SiC}-\mathrm{C}$.

[Table II - Composition of the matrix of zero-cement refractory castables in the $\mathrm{Al}_{2} \mathrm{O}_{3}-\mathrm{SiC}$-C system.]

\begin{tabular}{lcc}
\hline Matérias-primas & \%-volume & \%-peso \\
\hline $\mathrm{Al}_{2} \mathrm{O}_{3}$ calcinada A-3000FL & 50,28 & 52,16 \\
\hline $\mathrm{SiC}$ & 12,22 & 10,21 \\
\hline grafite & 2,70 & 1,46 \\
\hline $\mathrm{Al}_{2} \mathrm{O}_{3}$ eletrofundida & \\
branca 200/F* & 34,80 & 36,17 \\
\hline teor de sólidos & 62,9 & 86,6 \\
\hline
\end{tabular}

* fração com granulometria inferior a $44 \mu \mathrm{m}$ (\#325).

$\mathrm{C}_{16} \mathrm{H}_{33}\left(\mathrm{OCH}_{2} \mathrm{CH}_{2}\right)_{\mathrm{n}} \mathrm{OH}$ - polyoxyethylene $(\mathrm{n}=20)$ cetyl ether (Sigma Aldrich Química, $1120 \mathrm{~g} / \mathrm{mol}$ ).

A eficiência dos aditivos, ácido cítrico e Darvan-7S, na dispersão das suspensões representativas da matriz de concretos contendo $0,2 \mathrm{mg} / \mathrm{m}^{2}$ de surfactante (teor otimizado através de ensaios preliminares), foi avaliada através de medidas de viscosidade em função do teor de aditivo (expresso como a razão massa do aditivo/ área específica das partículas). As medidas de viscosidade foram efetuadas em um equipamento digital Brookfield modelo LVDVIII, utilizando um acessório para pequenas amostras (SC4).

O comportamento reológico de suspensões representativas da matriz (62,9\% vol. sólidos) contendo $0,2 \mathrm{mg} / \mathrm{m}^{2}$ de surfactante, foi avaliado através de medidas de viscosidade e tensão de escoamento em função do $\mathrm{pH}$ para diferentes teores de ácido cítrico. Tais medidas foram obtidas através da aplicação de um ciclo de cisalhamento utilizando as seguintes taxas: 2,$5 ; 5,0 ; 7,5 ; 10 ; 20$; $30 ; 40$ e $50 \mathrm{~s}^{-1}$, com tempo de permanência de $20 \mathrm{~s}$ em cada rotação. Os ajustes de $\mathrm{pH}$ foram realizados com a adição de solução aquosa de $\mathrm{HNO}_{3}$ ou $\mathrm{KOH}$, após a adição de uma quantidade fixa de ácido cítrico. As medidas de viscosidade aparente foram avaliadas à taxa de cisalhamento de $50 \mathrm{~s}^{-1}$, em virtude da sua correlação direta com a fluidez dos concretos [5]. A tensão de escoamento, por sua vez, foi determinada ajustando-se os dados experimentais ao modelo de Casson [11]. De acordo com esse modelo (equação A), os valores 
de tensão de escoamento $\left(\tau_{\mathrm{o}}\right)$ equivalem ao quadrado do coeficiente linear da reta obtida no gráfico de $\tau^{1 / 2}$ em função da $\dot{\gamma}^{1 / 2}$.

$$
\tau^{1 / 2}=\tau_{\mathrm{o}}^{1 / 2}+\left(\eta_{\dot{\gamma}}\right)^{1 / 2}
$$

onde $\eta$ é a viscosidade; $\tau$ e $\dot{\gamma}$ são a tensão e a taxa de cisalhamento.

Os resultados de viscosidade e de tensão de escoamento foram utilizados para construir mapas de estabilidade da matriz para concretos zero-cimento no sistema $\mathrm{Al}_{2} \mathrm{O}_{3}-\mathrm{SiC}-\mathrm{C}$. A eficiência de tais mapas em prever as condições ótimas de dispersão da matriz foi verificada através de medidas de $\mathrm{pH}$ e fluidez de concretos zerocimento com diferentes teores de ácido cítrico. A composição dos concretos é apresentada na Tabela III. Embora esta composição não seja aquela normalmente usada em canais de corrida, a relação percentual entre as matérias-primas que compõem os finos respeita as composições industriais. A distribuição de tamanho de partículas foi ajustada para uma curva teórica de acordo com o modelo de Andreasen com q=0,21. Nenhum ligante hidráulico ou cimento foi adicionado para simplificar a avaliação do efeito da dispersão e da distribuição granulométrica sobre o comportamento reológico das suspensões. A preparação das composições envolveu a homogeneização das matérias-primas em um misturador planetário (Pavitest modelo C3010) por $30 \mathrm{~s}$ e posterior adição gradativa de água contendo $0,2 \mathrm{mg} / \mathrm{m}^{2}$ do surfactante não-iônico. Após ser atingido o ponto da virada do concreto, continuou-se a mistura por mais 3 minutos. $\mathrm{O}$ teor de água dos concretos foi mantido fixo em 16,8\%-vol. (teor necessário para a virada), que corresponde a um teor de água na matriz de 37,1\%-vol. (62,9\% em volume de sólidos).

Um teste de fluidez livre ("free-flow") adaptado da norma ASTM C-860 foi usado para obter um índice de fluidez do concreto. Combinações de teor de ácido cítrico e $\mathrm{pH}$, bem como os dados de fluidez obtidos, foram adicionados ao mapa de estabilidade e comparados com a região ótima de dispersão. Ajustes do $\mathrm{pH}$ do

Tabela III - Composição de concreto zero-cimento no sistema $\mathrm{Al}_{2} \mathrm{O}_{3}$-SiC-grafite.

[Table III - Composition of the zero-cement refractory castables in the $\mathrm{Al}_{2} \mathrm{O}_{3}-\mathrm{SiC}-\mathrm{C}$ system.]

\begin{tabular}{lccc}
\hline Matérias-primas & & \%-volume & \%-peso \\
\hline \multirow{2}{*}{ Alumina } & $4 / 10$ & 17,29 & 17,44 \\
eletrofundida & $8 / 20$ & 9,15 & 9,27 \\
branca & $10 / 36$ & 8,14 & 8,22 \\
(agregado) & $20 / 40$ & 7,12 & 7,24 \\
& $40 / \mathrm{F}$ & 9,15 & 9,26 \\
& $60 / \mathrm{F}$ & 9,15 & 9,33 \\
& $200 / \mathrm{F}$ & 17,38 & 17,81 \\
Finos & $\mathrm{Al} \mathrm{O}_{3}$ calcinada & 16,75 & 17,12 \\
\hline & SiC & 4,07 & 3,35 \\
& Grafite como & 1,80 & 0,96 \\
\hline & recebido & & \\
\hline
\end{tabular}

concreto, através do deslocamento para a região ácida com a adição de solução aquosa de $\mathrm{HNO}_{3}$, foram acompanhados de medidas de fluidez.

\section{RESULTADOS E DISCUSSÃO}

A avaliação da viscosidade e $\mathrm{pH}$ de suspensões representativas da matriz de concretos refratários zero-cimento no sistema $\mathrm{Al}_{2} \mathrm{O}_{3}$ SiC-C, em função do teor de aditivo é apresentada na Fig. 1.

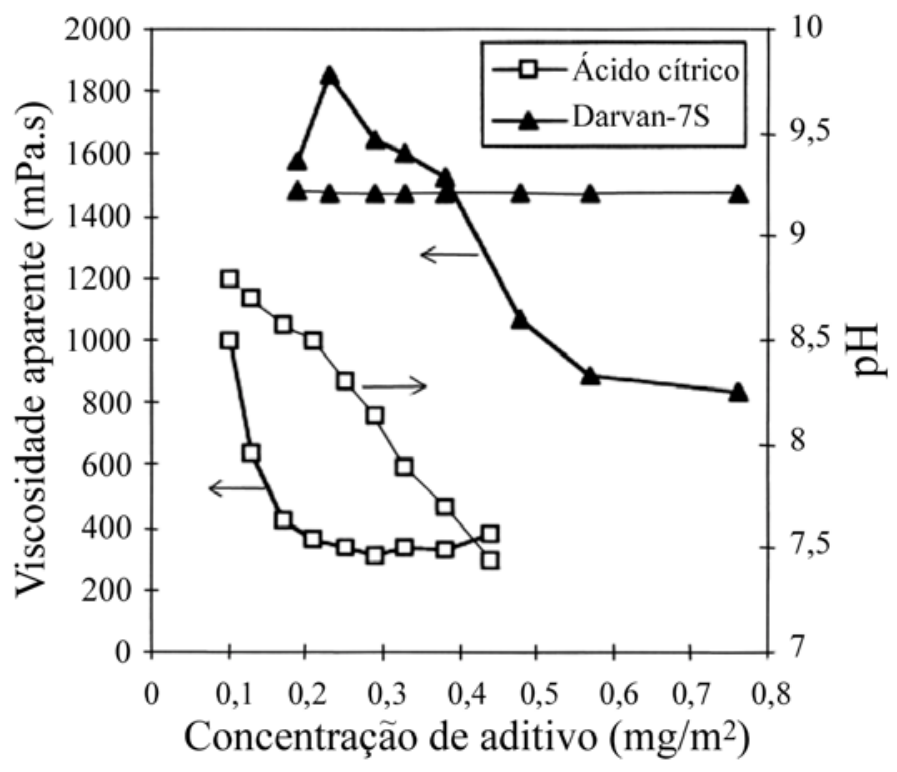

Figura 1: Variação da viscosidade e do $\mathrm{pH}$ em função da concentração de ácido cítrico e Darvan-7S para suspensões representativas da matriz de concreto refratário zero-cimento no sistema $\mathrm{Al}_{2} \mathrm{O}_{3}-\mathrm{SiC}-\mathrm{C}$, contendo $62,9 \%$ em volume de sólidos e $0,20 \mathrm{mg} / \mathrm{m}^{2}$ de surfactante.

[Figure 1: Apparent viscosity and pH values, as a function of polyacrylic and citric acids concentration, for the matrix suspensions of zero-cement refractory castables in the $\mathrm{Al}_{2} \mathrm{O}_{3}-\mathrm{SiC}$-C system, containing 62.9 vol\% solids and $0.20 \mathrm{mg} / \mathrm{m}^{2}$ of surfactant.]

Os aditivos Darvan-7S e ácido cítrico são caracterizados como dispersantes anionicos e se diferenciam quanto ao tipo de estrutura. O Darvan-7S apresenta uma estrutura polimérica enquanto o ácido cítrico apresenta uma estrutura molecular fixa. Ambos apresentam em suas estruturas químicas grupos $\mathrm{COOH}$ como grupos funcionais, cuja dissociação é favorecida em meio básico (reação B). Quando adsorvidos na superfície das partículas, as cargas negativas dos aditivos promovem repulsão entre as mesmas resultando na dispersão das suspensões.

$$
\mathrm{RCOOH}+\mathrm{H}_{2} \mathrm{O} \stackrel{\mathrm{OH}^{-}}{\longrightarrow} \mathrm{RCOO}^{-}+\mathrm{H}_{3} 0^{+}
$$

Como pode ser observado na Fig. 1 a adição de ácido cítrico mostra-se mais eficiente na dispersão do sistema $\mathrm{Al}_{2} \mathrm{O}_{3}-\mathrm{SiC}-\mathrm{C}$, embora promova um decréscimo no $\mathrm{pH}$ da suspensão. As suspensões utilizadas nesses ensaios continham um teor fixo de surfactante, necessário para promover a molhabilidade das partículas de grafite e, assim, possibilitar a homogeneização da suspensão. Isso é possível pelo fato dos surfactantes associarem em uma mesma molécula 
grupos funcionais que apresentam elevada afinidade pelo meio líquido e grupos compatíveis com a superfície do sólido. Essas características possibilitam reduzir a energia interfacial entre sólidos hidrofóbicos (como o grafite) e a água, atenuando a tendência de aglomeração desses materiais em meio aquoso.

A superior eficiência do ácido cítrico em relação ao Darvan-7S provavelmente deve-se à possível ocorrência dos efeitos "depletion" e "bridging" nas suspensões contendo o polímero. De acordo com alguns pesquisadores [12], estes fatores afetam diretamente a interação entre as partículas em suspensão quando o comprimento molecular dos aditivos presentes na solução torna-se maior ou similar ao espaçamento interparticular. Esta condição pode ser preferencialmente encontrada em suspensões com alto teor de sólidos como é o caso dos concretos refratários.

O efeito "depletion" de atração é um fenômeno que ocorre devido a diferença na concentração de moléculas não adsorvidas na região entre as partículas $\left(\mathrm{C}_{\mathrm{i}}\right)$ e na solução restante $\left(\mathrm{C}_{\mathrm{b}}\right)$. Quando o comprimento da molécula é similar ou maior do que o espaçamento interparticular, as moléculas não conseguem entrar na região entre as partículas fazendo com que $\mathrm{C}_{\mathrm{i}}<\mathrm{C}_{\mathrm{b}}$, originando uma pressão osmótica que acaba por expulsar as moléculas existentes entre as partículas originando uma força de atração entre elas. O efeito "bridging", por sua vez, ocorre quando a mesma molécula em solução adsorve simultaneamente em duas partículas distintas, formando uma "ponte" entre elas.

As moléculas de ácido cítrico apresentam comprimento bem menor do que as cadeias de poliacrilato. Isso reduz a chance de ocorrência dos efeitos "depletion" e "bridging" quando ácido cítrico é utilizado na dispersão de suspensões com alto teor de sólidos [12].

\section{Comportamento Reológico da Matriz}

A composição da suspensão representativa da matriz (Tabela II) foi determinada de maneira a manter a proporção entre as matériasprimas finas utilizadas em composições de concretos para canal de corrida ( $74 \% \mathrm{Al}_{2} \mathrm{O}_{3}-18 \% \mathrm{SiC}-8 \%$ grafite, em volume). As matériasprimas $\mathrm{SiC}$ e grafite contribuem para o decréscimo do $\mathrm{pH}$ das suspensões devido às suas características ácidas. No caso dos concretos, o decréscimo do $\mathrm{pH}$ é menos acentuado devido à contribuição das demais matérias-primas constituintes do agregado, principalmente da alumina tipo 200/F. Dessa forma, a fração da alumina eletrofundida branca 200/F, com granulometria inferior a $44 \mu \mathrm{m}$ ( $67 \%$ do total), também foi considerada como constituinte da matriz, com o objetivo de aproximar as condições iniciais de $\mathrm{pH}$ do concreto e das suspensões. Uma diferença de $\mathrm{pH}$ inicial pode acarretar adições de quantidades distintas de ácido ou base às suspensões e ao concreto para um determinado ajuste de $\mathrm{pH}$. Neste caso, ocorreria uma acentuada diferença na força iônica do meio das suspensões e concretos, influenciando suas propriedades reológicas.

Tem sido recentemente proposto [5] que a viscosidade aparente, a tensão de escoamento e as características não-newtonianas da matriz, avaliadas por medidas de área de histerese, são os principais parâmetros reológicos que determinam a fluidez de concretos de alta alumina. Esses parâmetros foram avaliados variando-se o pH de suspensões representativas da matriz contendo teores fixos de ácido cítrico. As características não-newtonianas das suspensões apresentaram comportamento similar a tensão de escoamento. Dessa forma, somente os resultados de viscosidade e tensão de escoamento são apresentados na Fig. 2.
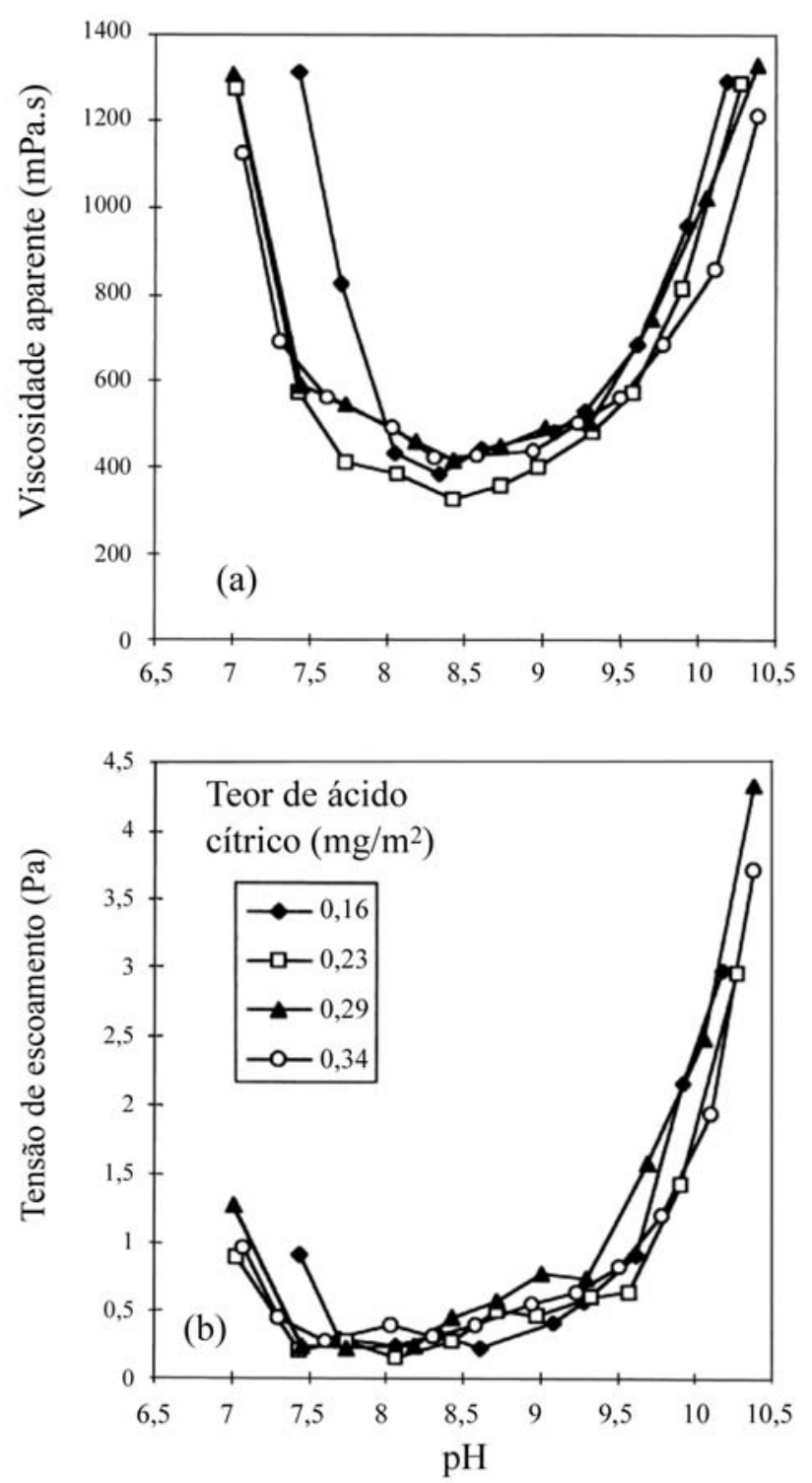

Figura 2: Curvas de viscosidade aparente (a) e tensão de escoamento (b) de suspensões representativas da matriz do concreto (62,9\%-vol.) em função do $\mathrm{pH}$ para diferentes teores de ácido cítrico.

[Figure 2: Apparent viscosity (a) and yield stress (b) for the matrix suspensions of zero-cement refractory castables in the $\mathrm{Al}_{2} \mathrm{O}_{3}$-SiC-C system (62.9 vol\%), as a function of $\mathrm{pH}$ for several citric acid contents.]

Na Fig. 2 verifica-se um aumento da viscosidade e tensão de escoamento, nos valores extremos de $\mathrm{pH}$ avaliados para qualquer teor de ácido cítrico, o que evidencia a ocorrência de aglomeração das partículas nessas condições. Uma vez que as suspensões são constituídas principalmente por $\mathrm{Al}_{2} \mathrm{O}_{3}$, pode-se supor que a interação entre $\mathrm{o} \mathrm{Al}_{2} \mathrm{O}_{3}$ e o ácido cítrico, controle o comportamento reológico das suspensões da matriz. Na presença de ácido cítrico o ponto isoelétrico das partículas de $\mathrm{Al}_{2} \mathrm{O}_{3}$ é deslocado para a região ácida $[5,8]$. Assim, com a adição de ácido às suspensões é verificado a formação de aglomerados fortes pela redução do potencial de repulsão entre as partículas. Por outro lado, com o deslocamento do $\mathrm{pH}$ da suspensão para a região básica, é verificado um acentuado decréscimo no teor de ácido cítrico adsorvido na superfície de $\mathrm{Al}_{2} \mathrm{O}_{3}$ [8]. Isso acarreta um aumento da força iônica do meio, possibilitando a formação de aglomerados fracos. A presença de aglomerados fracos que se quebram pela aplicação de taxas de cisalhamento 
relativamente baixas é a principal razão para a elevação da tensão de escoamento com o aumento do $\mathrm{pH}$.

\section{Mapas de Estabilidade e Medidas de Fluidez}

Mapas de estabilidade para concretos zero-cimento no sistema $\mathrm{Al}_{2} \mathrm{O}_{3}-\mathrm{SiC}-\mathrm{C}$, parametrizados pela viscosidade aparente e tensão de escoamento são apresentados na Fig. 3. Tem sido verificado que os mapas de estabilidade são ferramentas úteis para a previsão das condições de processamento (p.e., teor de aditivo e $\mathrm{pH}$ ), que originam suspensões estáveis [5]. Por exemplo, no mapa parametrizado pela viscosidade, toda combinação teor de aditivo-
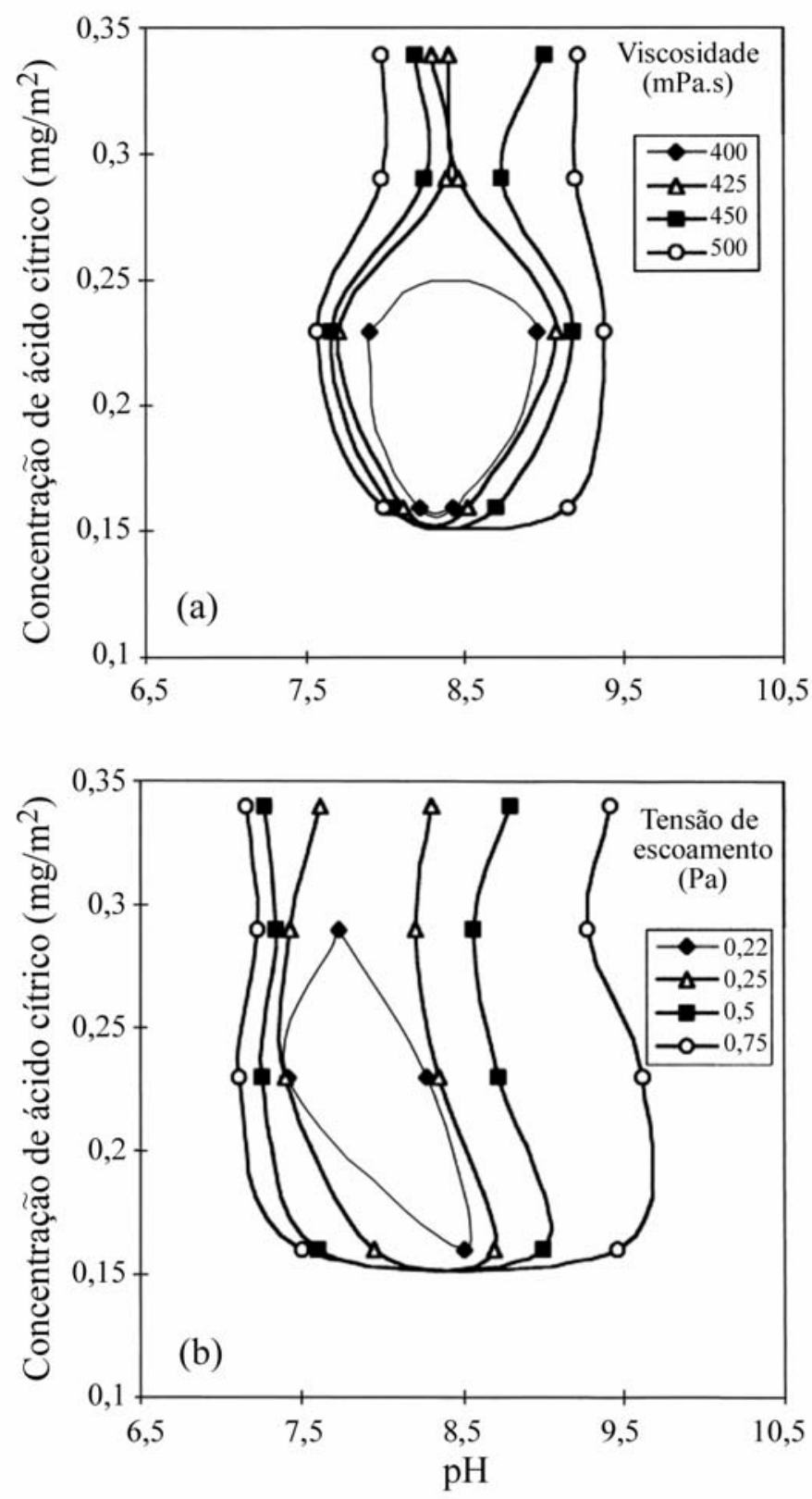

Figura 3: Mapas de estabilidade para suspensões representativas da matriz do concreto $(62,9 \%$-vol.) obtidos dos resultados de viscosidade aparente (a) e tensão de escoamento (b).

[Figure 3: Stability maps for the matrix suspensions of zero-cement refractory castables in the $\mathrm{Al}_{2} \mathrm{O}_{3}-\mathrm{SiC}$-C system (62.9 vol\%) obtained from the (a) apparent viscosity and the (b) yield stress measurements.]
pH no interior de uma certa linha deve originar suspensões com viscosidade menor do que o valor que essa linha representa.

$\mathrm{O}$ mapa de estabilidade para o sistema $\mathrm{Al}_{2} \mathrm{O}_{3}-\mathrm{SiC}-\mathrm{C}$ foi comparado com o mapa do sistema contendo apenas alumina [5], com relação à região ótima para a dispersão (linhas de isoviscosidade de 400 e $365 \mathrm{mPa} . \mathrm{s}$, respectivamente).

Observa-se que a faixa de teores de ácido cítrico referentes à região ótima do mapa para o sistema $\mathrm{Al}_{2} \mathrm{O}_{3}-\mathrm{SiC}-\mathrm{C}$ é sensivelmente inferior àquela correspondente às condições ideais de dispersão para o sistema contendo apenas alumina. Tendo em vista que as partículas de $\mathrm{SiC}$ apresentam cargas elétricas negativas em uma larga faixa de $\mathrm{pH}$ ( 4-12) [9], supõe-se que os ânions de citrato adsorvam preferencialmente na superfície das partículas de alumina, nas quais a densidade superficial de cargas positivas é significativamente maior. Em virtude disso, a região ótima do mapa referente ao sistema $\mathrm{Al}_{2} \mathrm{O}_{3}-\mathrm{SiC}-\mathrm{C}$ foi também determinada assumindo-se que todo o teor de ácido cítrico adicionado adsorva preferencialmente sobre as partículas de alumina. Neste caso, o teor de dispersante foi calculado com base apenas na área superficial das aluminas. A Fig. 4 mostra que, adotando esse procedimento de cálculo, a região ideal para a dispersão do sistema $\mathrm{Al}_{2} \mathrm{O}_{3}-\mathrm{SiC}-\mathrm{C}$ se aproxima bastante da região ótima referente às suspensões contendo apenas alumina. Tais resultados indicam que o comportamento reológico das suspensões $\mathrm{Al}_{2} \mathrm{O}_{3}-\mathrm{SiC}-\mathrm{C}$ avaliadas é controlado principalmente pelo estado de dispersão das partículas de alumina. Além da adsorção preferencial de íons de citrato pelas partículas de alumina, o elevado teor desta matéria-prima nas suspensões estudadas também justifica os resultados obtidos.

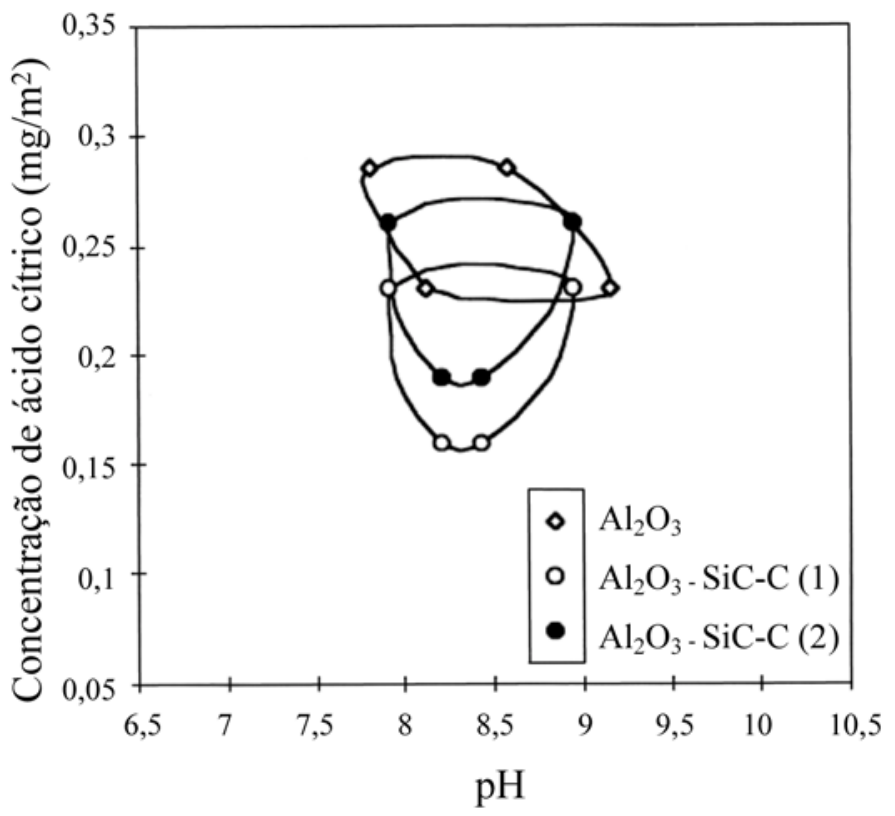

Figura 4: Mapas de estabilidade para o sistema contendo apenas $\mathrm{Al}_{2} \mathrm{O}_{3} \mathrm{e}$ para os sistemas $\mathrm{Al}_{2} \mathrm{O}_{3}$-SiC-C nos quais o teor de ácido cítrico foi calculado com base na área superficial de todas as matérias-primas (1) ou apenas na área superficial das aluminas A-3000FL e 200/F (2).

[Figure 4: Stability maps obtained from viscosity measurements for alumina suspensions (58 vol\%) and for suspensions containing $\mathrm{Al}_{2} \mathrm{O}_{3}, \mathrm{SiC}$ and $\mathrm{C}$ (62.9 vol\%), which the citric acid content was determined considering the specific area of all raw materials (1) or the specific area of aluminas $A$ 3000FL e 200/F (2).] 
Devido à dependência da fluidez do concreto em relação a viscosidade e tensão de escoamento da matriz, um mapa de estabilidade misto contendo as linhas de iso-viscosidade (400 e $450 \mathrm{mPa} . \mathrm{s})$ e iso-tensão $(0,22$ e $0,5 \mathrm{~Pa})$ é apresentado na Fig. 5. Neste mapa são apresentados os resultados de $\mathrm{pH}$ inicial e fluidez obtidos para concretos preparados com diferentes teores de ácido cítrico. Esses resultados mostram que as condições iniciais de $\mathrm{pH}$ do concreto não foram correspondentes à região ótima de dispersão estabelecida pelos mapas. O uso de elevados teores de ácido cítrico mostrou-se eficiente para otimizar a fluidez do concreto, uma vez que promove uma redução do $\mathrm{pH}$ para regiões do mapa de inferior viscosidade e tensão de escoamento. Da mesma forma, a adição de uma solução ácida a concretos contendo teores intermediários de ácido cítrico $\left(0,2 \mathrm{mg} / \mathrm{m}^{2}\right)$ também resulta em um aumento da fluidez do concreto, em decorrência do deslocamento do $\mathrm{pH}$ para valores próximos à região ótima de dispersão. Entretanto, ajustes de $\mathrm{pH}$ para valores mais ácidos mostraram um decréscimo na fluidez, apesar dos valores de $\mathrm{pH}$ ainda se encontrarem na faixa ótima do mapa. Isso pode ser explicado pela superior força iônica do concreto quando comparado às suspensões representativas nessas condições, uma vez que o $\mathrm{pH}$ da suspensão da matriz é inferior ao $\mathrm{pH}$ do concreto para qualquer teor de ácido cítrico.

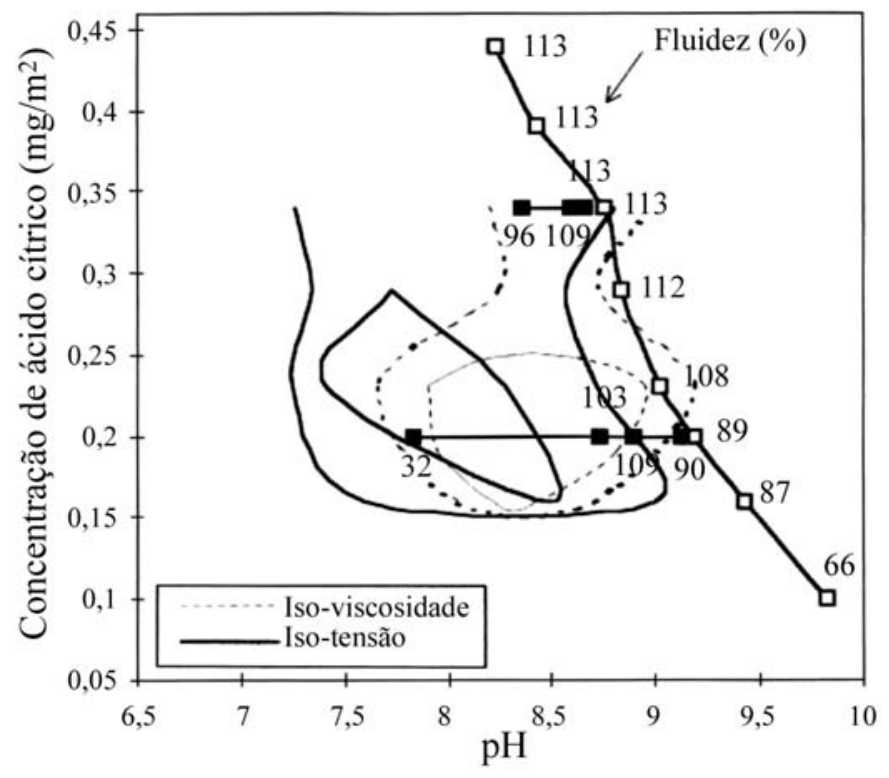

Figura 5: Mapa de estabilidade misto, o qual apresenta linhas de isoviscosidade e de iso-tensão; exibindo os resultados de fluidez e pH inicial para concretos preparados com diferentes teores de ácido cítrico. Para os teores de 0,2 e $0,34 \mathrm{mg} / \mathrm{m}^{2}$ de ácido cítrico também são apresentados os resultados de $\mathrm{pH}$ ajustados e respectivas medidas de fluidez.

[Figure 5: Mixed stability map, containing the iso-lines for viscosity (400 mPa.s) and yield stress ( $(0.22 \mathrm{~Pa})$, displaying the flowability and pH results of castables prepared at several citric acid contents. Flowability results and $\mathrm{pH}$ values for citric acid contents of 0.2 and $0.34 \mathrm{mg} / \mathrm{m}^{2}$ are also displayed.]

Concretos no sistema $\mathrm{Al}_{2} \mathrm{O}_{3}$-SiC-C também foram preparados substituindo-se grafite por coque. Os resultados de $\mathrm{pH}$ inicial e fluidez obtidos para esses concretos são apresentados na Fig. 6. Observa-se que os concretos preparados com coque apresentam maiores valores de fluidez para qualquer teor de ácido cítrico, quando comparado ao sistema $\mathrm{Al}_{2} \mathrm{O}_{3}$-SiC-grafite (Fig. 5). O grafite apresenta uma estrutura fechada com sítios ativos apenas ao redor das arestas do cristal, resultando numa alta resistência a corrosão e oxidação, porém reduzida molhabilidade. O coque, por sua vez, apresenta uma estrutura menos ordenada e consequentemente uma superior molhabilidade. Essa característica facilita o processamento desse material e faz com que ele seja a fonte de carbono normalmente utilizada em concretos comerciais. Para o sistema $\mathrm{Al}_{2} \mathrm{O}_{3}$-SiC-coque também é observado que a fluidez é otimizada pelo uso de altos teores de ácido cítrico e pela redução do $\mathrm{pH}$.

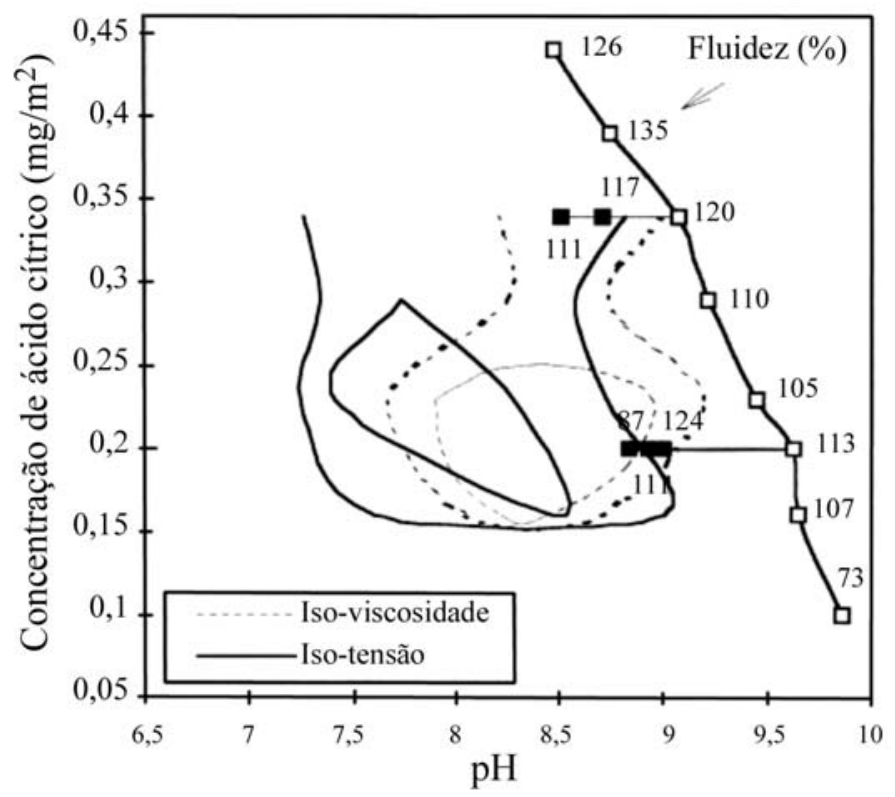

Figura 6: Mapa de estabilidade misto do sistema $\mathrm{Al}_{2} \mathrm{O}_{3}$-SiC-grafite, o qual apresenta os resultados de fluidez e $\mathrm{pH}$ inicial para concretos preparados com coque. Para os teores de 0,2 e $0,34 \mathrm{mg} / \mathrm{m}^{2}$ de ácido cítrico também são apresentados os resultados de $\mathrm{pH}$ ajustados e respectivas medidas de fluidez.

[Figure 6: Mixed stability map for the $\mathrm{Al}_{2} \mathrm{O}_{3}$-SiC-graphite system, displaying the flowability and $\mathrm{pH}$ results of castables prepared with coke. Flowability results and $\mathrm{pH}$ values for citric acid contents of 0.2 and 0.34 $\mathrm{mg} / \mathrm{m}^{2}$ are also displayed.]

\section{CONCLUSÕES}

A adição de moléculas orgânicas contendo grupos ionizáveis para promover a dispersão de suspensões cerâmicas mostrou-se muito eficiente. Quando sólidos hidrofóbicos como o grafite estão presentes na mesma suspensão, a dispersão pode ser otimizada pela adição conjunta de espécies surfactantes. Em virtude do alto teor de sólidos da matriz do concreto, aditivos de cadeia curta (como o ácido cítrico) tendem a ser mais eficientes, pelo fato de minimizarem a ocorrência dos efeitos "depletion" e "bridging".

O comportamento reológico da matriz de concretos refratários zero-cimento no sistema $\mathrm{Al}_{2} \mathrm{O}_{3}$-SiC-C é significativamente influenciado pelo $\mathrm{pH}$ e teor de ácido cítrico. Mapas de estabilidade para suspensões com alto teor de sólidos nesse sistema são apresentados neste trabalho, indicando as condições ótimas para a dispersão. A comparação dos mapas obtidos com o mapa de estabilidade referente a suspensões contendo apenas alumina indica 
que as partículas de alumina governam o comportamento reológico das suspensões $\mathrm{Al}_{2} \mathrm{O}_{3}$-SiC-C estudadas.

Observou-se ainda que as condições iniciais de $\mathrm{pH}$ dos concretos $\mathrm{Al}_{2} \mathrm{O}_{3}-\mathrm{SiC}-\mathrm{C}$ não são correspondentes à região ótima de dispersão estabelecida pelos mapas. A otimização da fluidez desses concretos (contendo grafite ou coque) requer a adição de elevados teores de ácido cítrico ou a adição de concentrações intermediárias desse dispersante juntamente com compostos que alterem o $\mathrm{pH}$ inicial para valores próximos à região ótima nos mapas de estabilidade obtidos. Embora não tenha sido observada uma correlação exata entre as condições de dispersão ótimas previstas e os valores de fluidez obtidos, os mapas de estabilidade propostos podem auxiliar significativamente a otimização do comportamento reológico dos concretos $\mathrm{Al}_{2} \mathrm{O}_{3}-\mathrm{SiC}-\mathrm{C}$.

\section{AGRADECIMENTOS}

Os autores agradecem à Alcoa Alumínio S.A. e à Unimetal pelo fornecimento das matérias-primas e à FAPESP pelo apoio recebido.

\section{REFERÊNCIAS}

[1] A. Nishikawa, "Technology of monolithic refractories", 1.ed. Tokyo: Plibrico Japan Company Ltd., (1984) 57-68.

[2] W. D. Boecker, "Silicon carbide: From Acheson's invention to new industrial products", cfi/Ber. DKG 74, 5 (1997) 244-251.

[3] W. Kenan, "Why graphite?", Ceram. Eng. Sci. Proc.,
16, 1 (1995) 178-180.

[4] C. F. Cooper, "Graphite, nature's unique raw material", J. Canadian Ceram. Soc. 63, 3 (1994) 197-208.

[5] A. R. Studart, W. Zhong, V. C. Pandolfelli, "Rheological Design of zero-cement self-flow castables", Am. Ceram. Soc. Bull. 78, 5 (1999) 65-72.

[6] K. Watanabe, M. Ishikawa, M. Wakamatsu, "Rheology of castable refractories", Taikabutsu Overseas 9, 1 (1989) 41-53.

[7] J. Cesarano III, I. A. Aksay, A. Bleier, "Stability of Aqueous $\alpha-\mathrm{Al}_{2} \mathrm{O}_{3}$ Suspensions with Poly(methacrylic acid) Polyelectrolyte", J. Am. Ceram. Soc. 71, 4 (1988) 250-255.

[8] P. Hidber, T. Graule, L. Gauckler, "Citric Acid-A Dispersant for Aqueous Alumina Suspensions”, J. Am. Ceram. Soc. 79, 7 (1996) 1857-1867.

[9] I. R. Oliveira, P. Sepulveda, V. C. Pandolfelli, "Heterodefloculação no sistema $\mathrm{Al}_{2} \mathrm{O}_{3}-\mathrm{SiC}$ ", Cerâmica 44, 290 (1998) 238-243.

[10] P. Somasundaran, S. Krishnakumar, "Adsorption of surfactants and polymers at the solid-liquid interface", Colloids and Surfaces. A: Physicochem. and Eng. Aspec. 123-124 (1997) 491-513.

[11] Lu-Cun. Guo et al. "Adsorption effects on the rheological properties of aqueous alumina suspensions with polyelectrolyte",

J. Am. Ceram. Soc. 81, 3 (1998) 549-556.

[12] A. R. Studart, J. Gallo, V. C. Pandolfelli, "Citric and polyacrylic acids as dispersants for high-alumina refractory castables", Aceito para publicação no Am. Ceram. Soc. Bull. (2001).

(Rec. 16/02/01, Ac. 22/06/01) 